Proyecciones

Vol. 23, No 1, pp. 15-29, May 2004.

Universidad Católica del Norte

Antofagasta - Chile

\title{
REGULAR CONDITIONAL PROBABILITY, DISINTEGRATION OF PROBABILITY AND RADON SPACES
}

\author{
D. LEAO Jr. ${ }^{\mathbf{a}}, M$. FRAGOSO ${ }^{\mathbf{b} *}$ and P. RUFFINO ${ }^{\dagger \mathbf{c}}$ \\ a Universidade de Sao Paulo, Brasil \\ b $L N C C$, Brasil \\ c Universidad Estadual de Campinas, Brasil
}

Received : September 2002. Accepted : November 2003.

\begin{abstract}
We establish equivalence of several regular conditional probability properties and Radon space. In addition, we introduce the universally measurable disintegration concept and prove an existence result.
\end{abstract}

Keywords : regular conditional probability, Radon space, separable Hausdorff measurable space, disintegration of probability.

AMS classification: 60A10, (93E20).

\footnotetext{
${ }^{*}$ Research supported, in part, by CNPq under grants $\mathrm{N} 520367 / 97-9$ and $\mathrm{N}$ 36.5532/2000-4, and PRONEX.

${ }^{\dagger}$ Written during a Sabbatical visit to the Mathematical Institute, University of Oxford, UK. Research supported by FAPESP grant No. 00/04591-3.
} 


\section{Introduction}

Since its inception, the concept of regular conditional probability (RCP), devised by Kolmogorov (1933), has been an important issue in probability theory. In 1948, in a celebrated paper, Dieudonné established the non existence of RCP for a suitable probability space. Since then, there has been a steadily increasing level of activity with RCP, which can be confirmed by the amount of papers in the specialized literature (see, e.g., Doob (1953), Sazonov (1965), Jirina (1954), Faden (1985), Leão, Fragoso and Ruffino (1999) and references therein). Besides the interest in its own right, the intervening years has shown an essential rôle played by this concept in a variety of applications (Bertsekas and Shreve (1978, chap. 10) and Getoor (1975), inter alia). The results range from existence and equivalence results to characterization via adequate spaces, including its liaison with some others important concepts.

Equivalence between properties of some RCP concepts was established in Faden (1985) for the case of separable probability space. In the context of separable probability space, existence of RCP is guaranteed via the property of either perfectness or compactness, see e.g. Hoffmann-Jörgensen (1971), Sazonov (1965) and Faden (1985). More recently we proved a necessary and sufficient condition for existence of RCP via an adequate characterization of the measurable space, independently of the probability on this space, see Leão, Fragoso and Ruffino (1999) and Leão (1999).

A related concept is the so called disintegration of a probability, introduced by von Neumann (1932) to study ergodic properties. This concept requires an additional difficulty of delimiting the support of the RCP. In a separable probability space existence conditions are analogous to that for RCP, described above (via, either perfectness or compactness). For further details see e.g. Hoffmann-Jörgensen (1971), Chatterji (1973), Valadier (1973) and Pachl (1978).

In this paper, going further in the characterization of Radon spaces, we establish equivalence among several related properties of RCP concepts, including necessary and sufficient conditions for its existence. Traditionally, the concept of probability disintegration is defined via measurability with respect to the complete $\sigma$-algebra. Motivated by applications in stochastic control with incomplete observations, we introduce the concept of universally measurable disintegration and establish sufficient conditions for its existence based on a suitable universally measurable selection result. Again, working only with $\sigma$-algebra properties, in the spirit of Leão, Fragoso and 
Ruffino (1999), our results are independent of the probability chosen for the measurable space. We conclude the paper with a slight extension of a disintegration result in Graf and Märgel (1980).

\section{Set up and preliminary results}

Let $(\Omega, \mathcal{F})$ be a measurable space. The atoms of $\mathcal{F}$ are the equivalence classes in $\Omega$ for the equivalence relation given by $w \sim w^{\prime}$ if and only if

$$
1_{A}(w)=1_{A}\left(w^{\prime}\right)
$$

for all $A \in \mathcal{F}$ where $1_{A}$ is the indicator function. The measurable space $(\Omega, \mathcal{F})$ is called Hausdorff if the atoms are the points of $\Omega$. If there exists a sequence of elements in $\mathcal{F}$ that generates $\mathcal{F}$ itself, we say the $\mathcal{F}$ is separable.

Given an abstract set $\Omega$ with a class of subsets $\mathcal{C}$ which contains the empty set and $\Omega$ itself, we say that $\mathcal{C}$ is a compact class if for every sequence $\left\{C_{n}: n \geq 1\right\}$ in $\mathcal{C}$ with intersection $\cap_{n \geq 1} C_{n}=\emptyset$ there exists an integer $n_{0}$ such that $\cap_{n \leq n_{0}} C_{n}=\emptyset$. For a probability space, we say that the triple $(\Omega, \mathcal{F}, P)$ is compact if there exists a compact class $\mathcal{C} \subset \mathcal{F}$ such that

$$
P(A)=\sup \{P(C): C \subset A, C \in \mathcal{C}\} \quad ; A \in \mathcal{F}
$$

For instance, if $(\Omega, \mathcal{F}, P)$ is a probability space where $\Omega$ is a topological space, $\mathcal{F}$ the Borel $\sigma$-algebra and $P$ a regular probability, then obviously $(\Omega, \mathcal{F}, P)$ is a compact probability space. In addition, a probability space $(\Omega, \mathcal{F}, P)$ is said to be perfect if for any measurable function $f: \Omega \rightarrow \mathbf{R}$ there exists a Borel set $B$ such that $B \subset f(\Omega)$ and $P\left[f^{-1}(B)\right]=1$. If the $\sigma$-algebra $\mathcal{F}$ is separable, it is well known that perfectness and compactness are equivalent, see e.g. Tortrat (1977, Proposition 3).

Given a measurable space $(E, \mathcal{E})$, we denote by $\mathcal{E}^{\lambda}$ the completion of $\mathcal{E}$ with respect to a probability $\lambda$ on this space. The universal $\sigma$-algebra $\mathcal{E}^{*}$ is defined by

$$
\mathcal{E}^{*}=\bigcap_{\lambda} \mathcal{E}^{\lambda}
$$

where the intersection is taken over all probabilities $\lambda$ on $(E, \mathcal{E})$.

Two measurable spaces are said to be isomorphic if there exists a bijection between them which is measurable and has a measurable inverse. Such a bijection is called a measurable isomorphism. Let $Y$ be a compact metric space endowed with its Borel $\sigma$-algebra $\beta(Y)$. We say that $(\Omega, \mathcal{F})$ is a Radon 
space if there exists a measurable isomorphism $\phi:(\Omega, \mathcal{F}) \rightarrow(U, \beta(U))$, where $U \in \beta(Y)^{*}$ and $\beta(U)=\{A \cap U: A \in \beta(Y)\}$, the trace of $\beta(Y)$ on $U$. Note that

$$
\beta(U)^{*}=\left\{B \cap U: B \in \beta(Y)^{*}\right\}
$$

The next theorem provides a characterization of Radon spaces which helps to understand this concept and to realise how rich the structure of this space is. It goes back to a result in our previous article and will be part of the proof of the main result of this paper, which, in fact, extends its scope.

Theorem 2.1 (Leão, Fragoso and Ruffino (1999)). A measurable space $(\Omega, \mathcal{F})$ is Radon if and only if it is separable, Hausdorff and for every probability $P$, the probability space $(\Omega, \mathcal{F}, P)$ is compact (or perfect).

Let $(\Omega, \mathcal{F}, P)$ be a probability space and $(E, \mathcal{E})$ a measurable space. A transition probability (or kernel) from $(E, \mathcal{E})$ to $(\Omega, \mathcal{F})$ is a function $\nu$ : $E \times \mathcal{F} \rightarrow[0,1]$ which satisfies the following two conditions:

a) $\nu(x, \cdot)$ is a probability on $\mathcal{F}$, for all $x \in E$;

b) $\nu(\cdot, A)$ is a measurable function on $(E, \mathcal{E})$, for all $A \in \mathcal{F}$.

Consider $T: \Omega \rightarrow E$ a measurable function. We say that $\nu: E \times \mathcal{F} \rightarrow$ $[0,1]$ is a $D$-transition probability ("D" here stands for Doob), if it satisfies (a) and

$\left.\mathrm{b}^{\prime}\right) \nu(\cdot, A)$ is a function measurable with respect to the complete $\sigma$-algebra $\mathcal{E}^{T_{*} P}$, for all $A \in \mathcal{F}$,

where $T_{*} P$ is the image probability of $T$ on $(E, \mathcal{E})$. We say that $\nu$ : $E \times \mathcal{F} \rightarrow[0,1]$ is a $U$-transition probability ("U" here stands for Universally measurable), if it satisfies (a) and

$\left.\mathrm{b}^{\prime \prime}\right) \nu(\cdot, A)$ is a function measurable with respect to the universally $\sigma$ algebra $\mathcal{E}^{\star}$, for all $A \in \mathcal{F}$.

Next, we define the six concepts of regular conditional probability which we shall consider in this paper; in fact these are the most commonly used in the literature. 
Definition 2.1. Let $(\Omega, \mathcal{F}, P)$ be a probability space and $(E, \mathcal{E})$ a measurable space,

1. RCP: Let $T: \Omega \rightarrow E$ be a measurable function. A regular conditional probability with respect to $T$ is a transition probability $\nu: E \times \mathcal{F} \rightarrow$ $[0,1]$ such that

$$
P\left[A \cap T^{-1}(B)\right]=\int_{B} \nu(x, A)\left(T_{*} P\right)(d x)
$$

for all $A \in \mathcal{F}$ and $B \in \mathcal{E}$;

2. Q-RCP: Let $f: \Omega \rightarrow \mathbf{R}$ be a measurable function. A quotient-RCP ent-RCP is an $R C P$ on the real line $\nu: \mathbf{R} \times \mathcal{F} \rightarrow[\mathbf{0}, \mathbf{1}]$;

3. P-RCP: Let $(\Omega \times E, \mathcal{F} \times \mathcal{E}, \lambda)$ be a product probability space. A product-RCP is a transition probability $\nu: E \times \mathcal{F} \rightarrow[0,1]$ satisfying

$$
\lambda(A \times B)=\int_{B} \nu(x, A) \lambda_{E}(d x)
$$

for all $A \in \mathcal{F}$ and $B \in \mathcal{E}$, where $\lambda_{E}$ is the marginal of $\lambda$ on $E$.

4. DQ-RCP: Let $f: \Omega \rightarrow \mathbf{R}$ be a measurable function. A D-quotient$R C P$ is a $D$-transition probability $\nu: \mathbf{R} \times \mathcal{F} \rightarrow[\mathbf{0}, \mathbf{1}]$ such that

$$
P\left[A \cap f^{-1}(B)\right]=\int_{B} \nu(x, A)\left(f_{*} P\right)(d x)
$$

for each $A \in \mathcal{F}$ and $B \in \beta(\mathbf{R})$

5. S-RCP: Let $\mathcal{E}$ be a sub- $\sigma$-algebra of $\mathcal{F}$, a subalgebra- $R C P$ is an $R C P$ $\nu$ from $(\Omega, \mathcal{E})$ to $(\Omega, \mathcal{F})$

6. D-RCP: Let $T: \Omega \rightarrow E$ be a measurable function. A $D$-RCP ient-RCP is a $D$-transition probability $\nu: E \times \mathcal{F} \rightarrow[0,1]$ such that

$$
P\left[A \cap T^{-1}(B)\right]=\int_{B} \nu(x, A)\left(T_{*} P\right)(d x)
$$

for each $A \in \mathcal{F}$ and $B \in \mathcal{E}$

Note that, since RCP concepts described above have emerged in different set ups, it is an interesting question in its own right to know how they are connected in a same space. For instance, the concept of RCP depends 
on the probability space $(\Omega, \mathcal{F}, P)$, the measurable space $(E, \mathcal{E})$ and the measurable function $T: \Omega \rightarrow E$. On the other hand, the concept of P-RCP depends on the measurable space $(E, \mathcal{E})$ and on the product probability $\lambda$, and the concept of S-RCP depends on the sub- $\sigma$-algebra $\mathcal{E}$. In order to compare these distinct regular conditional probability concepts, we follow Leão, Fragoso e Ruffino (1999) analysing these concepts on the measurable space $(\Omega, \mathcal{F})$.

We begin, in the spirit of what is done in Faden (1985), by introducing the properties which turns out to be all equivalent on a $\operatorname{Radon}$ space $(\Omega, \mathcal{F})$.

Definition 2.2. Let $(\Omega, \mathcal{F})$ be a measurable space.

1. RCPP: $(\Omega, \mathcal{F})$ has the $R C P$ property $P$, if for any probability $P$ on $(\Omega, \mathcal{F})$, any measurable space $(E, \mathcal{E})$ and any measurable function $T: \Omega \rightarrow E$ there exists a $R C P$;

2. Q-RCPP: $(\Omega, \mathcal{F})$ has the quotient-RCPP, if for any probability $P$ on $(\Omega, \mathcal{F})$ and any measurable function $f: \Omega \rightarrow \mathbf{R}$ there exists a $Q-R C P$;

3. P-RCPP: $(\Omega, \mathcal{F})$ has the product-RCPP, if for any probability $P$ on $(\Omega, \mathcal{F})$ and any product probability space $(\Omega \times E, \mathcal{F} \times \mathcal{E}, \lambda)$ for which $P$ is the $\Omega$-marginal, there exists a $P-R C P$;

4. DQ-RCPP: $(\Omega, \mathcal{F})$ has the $D$-quotient-RCPP, if for any probability $P$ on $(\Omega, \mathcal{F})$ and any measurable function $f: \Omega \rightarrow \mathbf{R}$, there exists a $D Q-R C P$;

5. S-RCPP: $(\Omega, \mathcal{F})$ has the subalgebra-RCPP, if for any sub- $\sigma$-algebra $\mathcal{E} \subset \mathcal{F}$ and any probability $P$ on $(\Omega, \mathcal{F})$, there exists a $S-R C P \nu(\cdot, \cdot)$ such that the probability space $(\Omega, \mathcal{F}, \nu(\omega, \cdot))$ is compact for all $\omega \in \Omega$.

6. D-RCPP: $(\Omega, \mathcal{F})$ has the $D-R C P P$ bra-RCPP, if for any probability $P$ on $(\Omega, \mathcal{F})$, any measurable space $(E, \mathcal{E})$ and any measurable function $T: \Omega \rightarrow E$, there exists a $D-R C P$;

It is clear that (1) implies (2) and (2) implies (4). Furthermore, we can establish that (3) implies (1). In fact, let $(\Omega, \mathcal{F})$ be a measurable space that satisfies the P-RCPP. Consider $P$ a probability on $(\Omega, \mathcal{F}),(E, \mathcal{E})$ a measurable space and $T: \Omega \rightarrow E$ a measurable function. Then, we obtain a probability $\lambda$ on the product space $(\Omega \times E ; \mathcal{F} \times \mathcal{E})$ such that

$$
\lambda(A \times B)=P\left[A \cap T^{-1}(B)\right]
$$


for each $A \in \mathcal{F}$ and $B \in \mathcal{E}$. Since $(\Omega, \mathcal{F})$ satisfies the P-RCPP, there exists a transition probability $\nu: E \times \mathcal{F} \rightarrow[0,1]$ satisfying

$$
P\left[A \cap T^{-1}(B)\right]=\lambda(A \times B)=\int_{B} \nu(x, A) \lambda_{E}(d x)
$$

where $\lambda_{E}(B)=\lambda(\Omega \times B)=P\left[T^{-1}(B)\right], A \in \mathcal{F}$ and $B \in \mathcal{E}$. Next, we shall establish the equivalence between these properties on Radon spaces.

\section{Main Results}

In this section, we shall establish connection between the various RCP properties and Radon spaces, introduce the universally measurable disintegration concept and prove an existence result.

Theorem 3.1. For a separable Hausdorff measurable space $(\Omega, \mathcal{F})$ the following statements are equivalents:

1) $(\Omega, \mathcal{F})$ is a Radon space;

2) for every probability $P$ on $(\Omega, \mathcal{F})$, the probability space $(\Omega, \mathcal{F}, P)$ is compact;

3) for every probability $P$ on $(\Omega, \mathcal{F})$, the probability space $(\Omega, \mathcal{F}, P)$ is perfect;

4) $(\Omega, \mathcal{F})$ satisfies the $R C P P$;

5) $(\Omega, \mathcal{F})$ satisfies the $Q-R C P P$;

6) $(\Omega, \mathcal{F})$ satisfies the $P-R C P P$;

7) $(\Omega, \mathcal{F})$ satisfies the $D Q-R C P P$;

8) $(\Omega, \mathcal{F})$ satisfies the $S-R C P P$;

9) $(\Omega, \mathcal{F})$ satisfies the $D-R C P P$;

Proof: From Theorem 2.1 we have that (1), (2) and (3) are equivalent. Equivalence of (1) and (4) is the content of Theorem 3.2 in Leão, Fragoso and Ruffino (1999). By Faden (1985, Theorem 6) we have that (3), (5), (6), and (7) are equivalent.

To prove that (2) is equivalent to (8), let $P$ be a (compact) probability on the Radon space $(\Omega, \mathcal{F})$ and $\mathcal{E} \subset \mathcal{F}$ a sub- $\sigma$-algebra. Then, there exists 
a S-RCP $\nu$ from $(\Omega, \mathcal{E})$ to $(\Omega, \mathcal{F})$. Since $\nu(x, \cdot)$ is a probability and $(\Omega, \mathcal{F})$ satisfies $(2)$, it implies that the probability space $(\Omega, \mathcal{F}, \nu(w, \cdot))$ is compact for all $\omega$.

On the other hand, suppose that for each probability $P$ on $(\Omega, \mathcal{F})$ and each sub- $\sigma$-algebra $\mathcal{E} \subset \mathcal{F}$ there exists an S-RCP $\nu$ such that the probability space $(\Omega, \mathcal{F}, \nu(w, \cdot))$ is compact for all $w \in \Omega$. In particular, if we take $\mathcal{E}=\{\emptyset, \Omega\}$, we obtain that $P(A)=\nu(w, A)$ for every $A \in \mathcal{F}$ and $w \in \Omega$. Hence $P$ is compact.

Since (4) implies (9) and (9) implies (7), it concludes the proof.

Let $(\Omega, \mathcal{F})$ be a Radon space with $T:(\Omega, \mathcal{F}) \rightarrow(E, \mathcal{E})$ a measurable function, where $(E, \mathcal{E})$ is a separable Hausdorff measurable space. If $P$ is a probability on $(\Omega, \mathcal{F})$, then it follows from Theorem 3.1 that there exists a $\operatorname{RCP} \nu$ from $(E, \mathcal{E})$ to $(\Omega, \mathcal{F})$. The added difficulty in disintegration problem is that of delimiting the support of the probability $\nu(x, \cdot)$. The space $\Omega$ is partitioned into the fibers $\left\{T^{-1}(\{x\}): x \in E\right\}$. Hence the natural question which appears is if the probability $\nu(x, \cdot)$ is concentrated on $T^{-1}(\{x\})$ for all $x \in T(\Omega)$. In sequence, we shall establish necessary and sufficient conditions for the existence of such RCP.

Theorem 3.2. Consider $(\Omega, \mathcal{F})$ and $(E, \mathcal{E})$ Borel spaces and $f: \Omega \rightarrow E$ a measurable function. Let $P$ be a probability on $(\Omega, \mathcal{F})$, then, there is a $R C P$ $\nu$ from $(E, \mathcal{E})$ to $(\Omega, \mathcal{F})$ such that $\nu\left(x, f^{-1}(\{x\})\right)=1$ for each $x \in f(\Omega)$ if, and only if, we have

i. $f(\Omega) \in \mathcal{E}$,

ii. There exists a function $g: f(\Omega) \rightarrow \Omega$ which is measurable with respect to the trace $\sigma$-algebra $\beta(f(\Omega))$ and $(g(x), x) \in G(f)$ for all $x \in f(\Omega)$.

Proof: Assume that $(i)$ and $(i i)$ are valid, we shall prove that there is a RCP $\nu$ such that $\nu\left(x, f^{-1}(\{x\})\right)=1$ for each $x \in f(\Omega)$. It follows from Theorem 3.1 and Hoffmann-Jörgensen (1971, Theorem 2) there exists a RCP $\mu$ and $N \in \mathcal{E}$ with $\left(T_{*} P\right)(N)=0$, such that

$$
\mu\left(x, f^{-1}(\{x\})\right)=1 ; x \in N^{c}
$$

Using the function $g$, we can define for each $A \in \mathcal{F}$

$$
\nu(x, A)=\left\{\begin{array}{cc}
\mu(x, A) & ; \quad x \in N^{c} \\
\delta_{A}(g(x)) & ; \quad x \in N \cap f(\Omega)
\end{array}\right.
$$


where $\delta$ is the Dirac probability. Thus, we conclude that $\nu$ is a RCP such that $\nu\left(x, f^{-1}(\{x\})\right)=1$ for each $x \in f(\Omega)$.

The converse of the theorem is a composition of well known results from the literature. So, if $\nu(x, \cdot)$ is concentrated on $f^{-1}(\{x\})$ for each $x \in f(\Omega)$, we can define

$$
\mu(w, B)=\nu(f(w), B) ; w \in \Omega \text { and } B \in \mathcal{F},
$$

which is a $\mathrm{S}-\mathrm{RCP}$ such that $\mu(w, A)=1$ for each $w \in A \in f^{-1}(\mathcal{E})$. Then, it follows from Blackwell and Dubins (1975, Theorem 4) that there exists a function $g_{1}: \Omega \rightarrow \Omega$ such that $g_{1}^{-1}(\mathcal{F}) \subset f^{-1}(\mathcal{E})$ and $w \in A \in f^{-1}(\mathcal{E})$ implies that $g_{1}(w) \in A$. Thus,

$$
f\left[g_{1}(w)\right]=f(w) ; w \in \Omega .
$$

Moreover, using Blackwell (1955, Theorem 4 (b)) there exists a measurable function $h: E \rightarrow \Omega$ such that

$$
h[f(w)]=g_{1}(w) ; w \in \Omega .
$$

We obtain from equations (3.1) and (3.2) that

$$
f(\Omega)=\{y \in E: f[h(y)]=y\}
$$

which is a Borel measurable subset of $E$.

Furthermore, it follows from Hoffmann-Jörgensen (1971, Theorem 3) that the graph of $f$ satisfies

$$
G(f)=\{(w, f(w): w \in \Omega\} \in \mathcal{F} \times \mathcal{E}
$$

and the sections $[G(f)]_{x}=\{w \in \Omega:(w, x) \in G(f)\}$ satisfy

$$
[G(f)]_{x}=f^{-1}(\{x\})
$$

for all $x \in f(\Omega)$. Thus, we have that

$$
\nu\left[x,[G(f)]_{x}\right]=1 ; x \in f(\Omega)
$$

Then, it follows from Blackwell and Ryll-Nardzewski (1963, Theorem 2) that there is a selection function $g: f(\Omega) \rightarrow \Omega$ measurable with the respect to the trace $\sigma$-algebra $\beta(f(\Omega))=\{C \cap f(\Omega): C \in \beta(\mathbf{R})\}$ and $(g(x), x) \in$ $G(f)$ for each $x \in f(\Omega)$. 
In what follows, we shall introduce some elements necessary to define universally measurable disintegration. If $(\Omega, \mathcal{F})$ is a Radon space and $\mathcal{E} \subset \mathcal{F}$ $(\Omega=E)$ is a sub- $\sigma$-algebra of subsets of $\Omega$, such that $\mathcal{E}$ contains all points, then, it follows from Musial (1972, Corollaries 3 and 4), that there exists a S-RCP $\nu$ satisfying

$$
\nu(x, A)=\mathbb{1}_{A}(x) ; x \in \Omega ; A \in \mathcal{E}
$$

if, and only if, we have that $\mathcal{F}=\mathcal{E}$, for any probability $P$ on $(\Omega, \mathcal{F})$.

Now, consider $(E, \mathcal{E})$ a separable, Hausdorff measurable space and $T$ : $\Omega \rightarrow E$ a measurable function. If $P$ is a probability on $(\Omega, \mathcal{F})$, then, it follows from Theorem 3.1 and Hoffmann-Jörgensen (1971, Theorems 2 and 3) that the graphic of $T$ is measurable with respect to the product $\sigma$-algebra,

$$
G(T)=\{(w, T(w)): w \in \Omega\} \in \mathcal{F} \times \mathcal{E}
$$

and there exists a $\mathrm{RCP} \mu: E \times \mathcal{F} \rightarrow[0,1]$ such that

$$
\mu\left(x, T^{-1}(\{x\})\right)=1 ;\left(T_{*} P\right)-\text { a.e. }
$$

Furthermore, the image $T(\Omega)$ is measurable with respect to the complete $\sigma$-algebra $\mathcal{E}^{T_{*} P}$. Since $P$ is arbitrary, we conclude that $T(\Omega)$ is universally measurable.

In the sequence, we shall introduce the concept of universally measurable disintegration and we shall establish sufficient condition for its existence.

Definition 3.1. Consider $(\Omega, \mathcal{F})$ a Radon space, $(E, \mathcal{E})$ a separable, Hausdorff measurable space, $T: \Omega \rightarrow E$ a measurable function and $P$ a probability on $(\Omega, \mathcal{F})$. A universally measurable disintegration gration with respect to $T$ is a $U$-transition probability $\nu: E \times \mathcal{F} \rightarrow[0,1]$ such that

$$
P\left[A \cap T^{-1}(B)\right]=\int_{B} \nu(x, A)\left(T_{*} P\right)(d x)
$$

for all $A \in \mathcal{F}, B \in \mathcal{E}$, and

$$
\nu\left(x, T^{-1}(\{x\})\right)=1
$$

for all $x \in T(\Omega)$.

With these elements we can establish the following proposition. 
Proposition 3.1. Let $(\Omega, \mathcal{F})$ be a Radon space, $(E, \mathcal{E})$ a separable, Hausdorff measurable space and $T: \Omega \rightarrow E$ a measurable function. If there exists a universally measurable selection function $\phi: T(\Omega) \rightarrow \Omega$ such that $(\phi(x), x) \in G(T)$ for each $x \in T(\Omega)$, then, there exists an universally measurable disintegration $\nu$, for any probability $P$ on $(\Omega, \mathcal{F})$.

Proof: We know that there exists a $\mathrm{RCP} \mu$ and $N \in \mathcal{E}$ with $\left(T_{*} P\right)(N)=$ 0 , such that

$$
\mu\left(x, T^{-1}(\{x\})\right)=1 ; x \in N^{c}
$$

Using the function $\phi$, we can define for each $A \in \mathcal{F}$

$$
\nu(x, A)=\left\{\begin{array}{ccc}
\mu(x, A) \quad ; & x \in N^{c} \\
\delta_{A}(\phi(x)) & ; \quad x \in N \cap T(\Omega)
\end{array}\right.
$$

where $\delta$ is the Dirac probability. Since $T(\Omega)$ is universally measurable subset of $E$, we conclude that $\nu$ is a universally measurable disintegration.

Next, we shall establish sufficient condition for the existence of an universally measurable selection function $\phi: T(\Omega) \rightarrow \Omega$ such that $(\phi(x), x) \in$ $G(T)$ for each $x \in T(\Omega)$.

Let $\left\{A_{i \mid n}\right\}$ be a countable family of sets indexed by the set of finite integers, where $i \mid n$ represents a sequence $\left\{i_{1}, \cdots, i_{n}\right\}$. If $i$ denotes an infinite sequence of positive integers $\left(i \in \mathbf{N}^{\mathbf{N}}\right)$, the set

$$
A=\bigcup_{i \in \mathbf{N}} \mathbf{N} \bigcap_{n=1}^{\infty} A_{i \mid n}
$$

is then said to be obtained from the family $\left\{A_{i \mid n}\right\}$ by Souslin operation. If $\mathcal{D}$ is a class of sets in a given space, the class of sets obtained by applying this operation to countable families in $\mathcal{D}$ is called $\mathcal{D}$-Souslin.

Consider $Y$ be a compact metric space endowed with the Borel $\sigma$-algebra $\beta(Y)$. A subset $A$ of $Y$ is analytic if $A \in F(Y)$-Souslin, where $F(Y)$ denotes the class of closed subsets of $Y$. We say that $(\Omega, \mathcal{F})$ is a Souslin space if there exists a measurable isomorphism $\phi:(\Omega, \mathcal{F}) \rightarrow(A, \beta(A))$, where $A$ is an analytic subset of $Y$ and $\beta(A)=\{B \cap A: B \in \beta(Y)\}$, the trace of $\beta(Y)$ on $A$. Since every analytic set is universally measurable, each Souslin space is also a Radon space. Furthermore, our definition of Souslin space is equivalent with Dellacherie and Meyer's definition [(1978, Theorem 20, pp. 
$48)]$. As the measurable space $(\Omega, \mathcal{F})$ is Souslin, we can define a metrizable topology on $\Omega$, such that $\mathcal{F}$ coincide with the Borel $\sigma$-algebra [see, Blackwell (1955), Theorem 2]. We denote by $F(\Omega)$ the class of closed subsets of $\Omega$.

Lemma 3.1. Let $(\Omega, \mathcal{F})$ be a Souslin space, $(E, \mathcal{E})$ a measurable space and $G \in \mathcal{F} \times \mathcal{E}^{\star}$. Then,

a. $\pi_{E}[G] \in \mathcal{E}^{\star}$

$b$. There exists a function $\phi: \pi_{E}[G] \rightarrow \Omega$ which is universally measurable and $(\phi(x), x) \in G$ for all $x \in \pi_{E}[G]$.

where $\pi_{E}[G]$ denotes de canonical projection of $G$ on $E$.

Proof: Let $R$ be the class of the measurable rectangles $F \times B$, where $F \in F(\Omega)$ e $B \in \mathcal{E}^{\star}$. We shall show that $G$ is $R$-Souslin. Consider $\mathcal{C}$ the class of all $R$-Souslin sets whose the complement is also $R$-Souslin. Then, we have that $\mathcal{C}$ is a $\sigma$-algebra [Dellacherie e Meyer (1978), Theorem 12, pp. 43]. For every $F \in F(\Omega)$ and $B \in \mathcal{E}^{\star}$, we have that

$$
F \times B \in \mathcal{C} \text { and }(F \times B)^{c}=\left(F \times B^{c}\right) \cup\left(F^{c} \times E\right)
$$

Since $F^{c} \in F(\Omega)$-Souslin, there exists a sequence $\left\{F_{\left(i_{1}, \cdots, i_{n}\right)}:\left(i_{1}, \cdots, i_{n}\right) \in\right.$ $\left.\mathbf{N}^{\mathbf{n}}, \mathbf{n} \in \mathbf{N}\right\} \subset \mathbf{F}(\boldsymbol{\Omega})$ satisfying

$$
F^{c}=\bigcup_{i \in \mathbf{N}} \mathbf{N} \bigcap_{n=1}^{\infty} F_{\left(i_{1}, \cdots, i_{n}\right)}
$$

Then, we conclude that

$$
F^{c} \times E=\left[\bigcup_{i \in \mathbf{N}} \mathbf{N} \bigcap_{n=1}^{\infty} F_{\left(i_{1}, \cdots, i_{n}\right)}\right] \times E=\bigcup_{i \in \mathbf{N} \mathbf{N}} \bigcap_{n=1}^{\infty}\left[F_{\left(i_{1}, \cdots, i_{n}\right)} \times E\right]
$$

Since $F \times B^{c}$ and $F^{c} \times E$ are $R$-Souslin sets, we obtain that $(B \times F)^{c}$ is also $R$-Souslin set. On the other hand, the product $\sigma$-algebra is generate by rectangles in $R$. Then, we have that $\mathcal{F} \times \mathcal{E}^{\star} \subset \mathcal{C}$. Thus, the elements of $\mathcal{F} \times \mathcal{E}^{\star}$ are $R$-Souslin sets. It follows from Leese (1978, Theorem 5.5), that:

a. $\pi_{E}(G) \in \mathcal{E}^{\star}$

b. There exists a function $\phi: \pi_{E} \rightarrow \Omega$ which is universally measurable and $(\phi(x), x) \in G$ for each $x \in \pi_{E}[G]$. 
Finally, we use the above results to extend a disintegration theorem in Graf and Märgel (1980).

Corollary 3.1. Let $(\Omega, \mathcal{F})$ be a Souslin space and $(E, \mathcal{E})$ a separable, Hausdorff measurable space. Then, there exists an universally measurable disintegration $\nu$, for each measurable function $T: \Omega \rightarrow E$ and probability $P$ on $(\Omega, \mathcal{F})$.

ACKNOWLEDGEMENT: During the preparation of this paper the third author was a visiting fellow at the Mathematical Institute in the University of Oxford. He is grateful to the friendly hospitality of Prof. Terry Lyons. He is also thankful for the financial support by FAPESP, proc. no. 00/04591-3.

\section{References}

[1] Bertsekas D. P., Shreve S. E. - Stochastic Optimal Control: The Discrete-Time Case, Academic Press, New York, (1978).

[2] D. Blackwell - On a class of probability spaces, Proc. Third Berkeley Symp. Math. Statist. Prob., pp. 1-6, University of California Press, (1955).

[3] D. Blackwell and C. Ryll-Nardzewski - Non-existence of everywhere proper conditional distribuition, Ann. Math. Statistics, Vol. 34, pp. 223-225, (1963).

[4] D. Blackwell and L. E. Dubins - On existence and non-existence of proper, regular, conditional distributions, Annals Probability, Vol. 3, No. 5, pp. 741-752, (1975).

[5] S. D. Chatterji - Disintegration of measures and lifting, Vector and Operator Valued Measures and Applications, Eds. D. H. Tucker and H. B. Naymard (Proc. Symp. Alta, Utah, 1972, pp. 69-83), Academic Press, New York, (1973).

[6] J. Dieudonné - Sur le théorème de Lebesgue-Nikodym III, Ann. Univ. Grenoble, Vol. 23, pp. 25-53, (1948).

[7] J. L. Doob - Stochastic Processes, John Wiley ans Sons, INC., (1953). 
[8] A. M. Faden - The existence of regular conditional probabilities: necessary and sufficient conditions, Annals Probability, Vol. 13, no. 1, pp. 288-298, (1985).

[9] S. Graf and G. Märgel - Disintegration of a measure with respect to a correspondence, Lecture Notes in Math., Vol. 794, Springer-Verlag, pp. 167-169, (1980).

[10] P. K. Getoor - On the Construction of Kernels, Lecture Notes in Mathematics, Séminaire de Probabilités IX, Vol. 465, pp. 443-463, (1975).

[11] J. Hoffmann-Jörgensen - Existence of Conditional Probabilities, Math. Scand., Vol. 28, pp. 257-264, (1971).

[12] M. Jirina - Probabilités conditionnelles sur des algèbres à base dénombrable, Czechosloviak Math. J., Vol. 4, pp. 372-380, (1954).

[13] A. N. Kolmogorov - Grundbegreffi der Wahrscheinlichkeitsrechnung, Springer, Berlin, (1933).

[14] D. Leão - Radon spaces, Ph.D. thesis, State University of CampinasUNICAMP, Brazil, Nov. (1999).

[15] D. Leão, M. D. Fragoso and P. Ruffino - Characterization of Radon spaces, Statistics and Probability Letters, Vol. 42, pp. 409-413, (1999).

[16] S. J. Leese - Measurable selections and the uniformization of Souslin sets, American Journal of Mathematics, Vol. 1000, No. 1, pp. 19-41, (1978).

[17] K. Musial - Existence of proper conditional probabilities, $Z$. Wahrscheinlichkeitstheorie verw. Geb., Vol. 22, pp. 8-12, (1972)

[18] J. von Neumann - Zur operatorenmethode in der Klassischen Mechanik, Annals of Math., vol. 33, pp. 587-642, (1932).

[19] J. K. Pachl - Disintegration and compact measures, Math. Scand., Vol. 43, pp. 157-168, (1978).

[20] V. V. Sazonov - On perfect measures, Amer. Math. Soc. Transl. Series 2, Vol. 48, pp. 229-254, (1965).

[21] M. M. Valadier - Désintegration d'une mesure sur un produit, $C$. $R$. Acad. Sc. Paris, série A, vol. 276, pp. 33-35, (1973). 
D. Leao Jr.

Departamento de Ciência da Computaçao e Estatística

ICMC - USP

Brasil

e-mail : leao@icmc.sc.usp.br

Marcelo D. Fragoso

LNCC

Petrópolis

Río de Janeiro

Brasil

and

R. C. Ruffino

Departamento de Matemática

Universidade Estadual de Campinas

13081-970 - Campinas - SP

Brasil

e-mail : ruffino@ime.unicampo.br 